\title{
Genetic algorithms based approach for designing spring brake orthosis - Part II: Control of FES induced movement
}

\author{
M.S. Huq ${ }^{\mathrm{a}, *}$ and M.O. Tokhi ${ }^{\mathrm{b}}$ \\ ${ }^{a}$ Department of Mechanical and Aerospace Engineering, Carleton University, Ottawa, ON, Canada \\ ${ }^{\mathrm{b}}$ Department of Automatic Control and Systems Engineering, University of Sheffield, Sheffield, UK
}

\begin{abstract}
Spring brake orthotic swing phase for paraplegic gait is initiated through releasing the brake on the knee mounted with a torsion spring. The stored potential energy in the spring, gained from the previous swing phase, is solely responsible for swing phase knee flexion. Hence the later part of the SBO operation, functional electrical stimulation (FES) assisted extension movement of the knee has to serve an additional purpose of restoring the spring potential energy on the fly. While control of FES induced movement as such is often a challenging task, a torsion spring, being antagonistically paired up with the muscle actuator, as in spring brake orthosis (SBO), only adds to the challenge. Two new schemes are proposed for the control of FES induced knee extension movement in SBO assisted swing phase. Even though the control schemes are closed-loop in nature, special attention is paid to accommodate the natural dynamics of the mechanical combination being controlled (the leg segment) as a major role playing feature. The schemes are thus found to be immune from some drawbacks associated with both closed-loop tracking as well as open-loop control of FES induced movement. A leg model including the FES knee joint model of the knee extensor muscle vasti along with the passive properties is used in the simulation. The optimized parameters for the SBO spring are obtained from the earlier part of this work. Genetic algorithm (GA) and multi-objective GA (MOGA) are used to optimize the parameters associated with the control schemes with minimum fatigue as one of the control objectives. The control schemes are evaluated in terms of three criteria based on their ability to cope with muscle fatigue.
\end{abstract}

Keywords: Functional electrical stimulation, fuzzy logic control, genetic algorithm, hybrid orthosis system, multi-objective genetic algorithms, optimization, spring brake orthosis

\section{Introduction}

Control of functional electrical stimulation (FES) induced movement is a significantly challenging arena for researchers. The challenge mainly emanates from various characteristics of the underlying physiological/biomechanical system. Muscle response

*Corresponding author: M.S. Huq, Department of Mechanical and Aerospace Engineering, Carleton University, Ottawa, ON, Canada. E-mail: mshuq@ connect.carleton.ca. characteristics are nonlinear and time-varying. The response of stimulated muscle changes primarily as the muscle fatigues $[4,14]$. There may also be inherent variability present in the response of the stimulated muscle [36]. Also certain motor reflexes at the spinal cord level may be preserved in individuals with spinal cord injury (SCI). These reflexes are often unpredictable and may impede with joint movements. Spasticity is also common in SCI, and is characterized by varying degrees of increased muscle tone and hyperactive spinal reflexes [35]. In the absence of 
supra-spinal signals, muscles can develop a tendency to maximally contract in response to a wide range of muscular or cutaneous stimuli, causing the limbs to be in an abnormally flexed position. Besides, the neuromuscular system is a highly coupled system; for instance, the knee joint torque exerted by the quadriceps muscle is potentially a function of the knee joint and hip joint angles, among other factors. The significant time delay between stimulation and the onset of a muscle contraction, in addition to the processing and transmission delays involved in the electrical stimulation system contributes a great deal in making up the challenge from the movement control perspective.

Primarily due to the complexity of the system (nonlinearities, time-variations, limited actuator (muscle) band-width and power) practical FES systems are predominantly open-loop systems where the controller receives no information about the actual state of the system [7]. Practical success of this open-loop control strategy is still, however, seriously limited due to this fixed nature of the associated parameters. The problem arises especially due to the existing parameter variations (e.g. muscle potentiation and fatigue), inherent time-variance, time-delay, and strong nonlinearities present in the neuromuscular-skeletal system or the plant to be controlled. Veltink [38] and Franken et al. [13] investigated a discrete-time proportional-integralderivative (PID) feedback controller for cycle-to-cycle adaptation of an experimentally initialized stimulation signal with a view to compensate for the potentiationand fatigue-induced time variation of muscle output. Abbas and Chizeck [1] proposed the so called pattern generator/pattern shaper (PG/PS) control system to automatically adjust stimulation patterns to suit the needs of a particular individual as well as to reduce the effects of muscle fatigue on control system performance.

Accurate control of movement can be ensured with closed-loop adaptive control mechanism to tackle FES control problem. It has several advantages over openloop schemes, such as better tracking performance and smaller sensitivity to modelling errors, parameter variations, and external disturbances. So far classical closed-loop control algorithms have failed to provide satisfactory performance and have not been able to guarantee stability, a desired property of the controlled system [27]. Jaime et al. [26] and Matjacic et al. [30] implemented PID controllers for unsupported standing in paraplegic subjects. Hunt and co-workers used Hinfinity control $[22,24]$ and linear quadratic Gaussian control [16] for unsupported standing in paraplegic subjects. They reported stable standing and were able to reject a 1 degree perturbation about the ankle joint. Hatwell et al. [21] used a model reference controller for FES control of knee joint movement in paraplegics. Previdi and Carpanzano [33] used a gain scheduling control strategy that interpolated between locally valid linear quadratic regulators for controlling FES-induced knee joint movement. Ferrarin et al. [10] developed an adaptive control algorithm for FES-induced knee joint movement. The controller used an inverse dynamic model of the quadriceps muscle to deliver stimulation to both the muscle and a direct dynamic model of the muscle. The error between the measured and predicted knee angles drove the adaptation mechanism. Jezernik et al. [27] used sliding mode FES control to regulate knee joint angle. The controller was tested on six neurologically intact subjects and two untrained paraplegic subjects. Good tracking of a desired knee joint trajectory was achieved for up to 8 seconds.

The spring brake orthosis (SBO), a kind of hybrid orthosis system (HOS), was developed to assist paraplegic gait by generating the swing phase [15]. SBO features a torsion spring along with a brake mounted on the knee joint through an external orthosisand FES at quadriceps muscle. While the torsion spring is responsible for the knee flexion movement through releasing the potential energy stored in it during the initial part of the swing phase, FES of quadriceps extends the knee joint against the spring force back to full extension during the following part of the swing phase; feeding energy to the torsion spring simultaneously to restore its potential energy. Selection of the most suitable spring for the SBO has been presented in Huq and Tokhi [25].

The current study uses the best spring parameters values from Huq and Tokhi [25] and hence essentially is a continuation from there. New closed-loop FES control strategies based on PID and fuzzy logic controller (FLC) are proposed in this paper to control the latter half of the SBO assisted paraplegic swing phase, i.e. control of FES induced knee extension movement. Unlike existing FES control approaches, the primary motivation driving the proposed control strategies is to accommodate as well as exploit the natural dynamics of the plant with a view to reduce fatigue of the FES muscle; and is brought about either by using a reference trajectory coincident with the natural oscillation of the plant or by part-time closed-loop bang-bang control with only target joint orientation as the fixed 
reference and thus allowing the joint trajectory to naturally take its course. In addition to their ease of implementation given the type of the model used in this study, PID and FLC are chosen as the basic control blocks to include representatives from both linear and nonlinear controllers. In principle, the proposed control schemes are expected to be suitable not just for SBO but also for other FES control applications involving movement of cyclical nature. Stochastic search approaches like genetic algorithm (GA) and its variant multi-objective GA (MOGA) are particularly preferable to search for optimum parameters in situations where the model is black-box like, lacking mathematical description. Both GA and MOGA are used throughout this work to tune the controllers, and in some cases few other parameters of interest.

\section{Methods}

\subsection{The leg model}

Two degrees of freedom (DoF) forward dynamic planar model of a human leg of an average-sized person (75 kg mass and $177 \mathrm{~cm}$ height) is developed within Visual Nastran (vN4D) software. The thigh, shank and foot are implemented as 3 rigid bodies. As in the originally proposed SBO the ankle joint is restrained with ankle-foot-orthosis (AFO). In the vN4D model, the shank and foot segments are connected through a 'rigid joint' to effectively act like a single rigid body connected to the thigh segment through hinge joint in sagittal plane. This whole leg segment connects to and suspends from a fixed segment (presumably pelvis) through 'hip joint' simulated as another hinge joint in the sagittal plane. Anthropometric data for the segments viz. volume, mass, centre of mass (CoM), joint centre and moment of inertia were obtained from statistical tables based on person's height and weight [41].

The passive knee and hip joint properties model, developed by Amankwah et al. [2] is also implemented in the Simulink and incorporated with the segmental dynamics in vN4D. The model describes the lower limb passive joint moments (ankle, knee and hip), and is based on Kelvin model for viscoelasticity comprising a nonlinear elastic element in parallel with both a linear elastic element and a nonlinear viscous element in series.

The FES knee joint model also comprises muscle model for the monoarticular knee extensor muscle, vasti and its associated moment arm model, both from the work of Riener and Fuhr [34] (Fig. 1). As the muscle generates force in response to electrical stimulation, the resultant joint torque is obtained from the force being multiplied by the associated moment arm. To make the control operation easier, the muscle vasti, which refers to the combination of vastus lateralis, vastus intermedius and vastus medialis, was especially chosen because of its monoarticular nature, so that the stimulation only delivers torque at the knee joint, leaving the adjacent joints unaffected. Figure 2 shows the simplified schematic of the overall knee joint model.

The total knee joint torque is the sum of active (FES) and passive viscoelastic torque taken from the work of Amankwah et al. [2]. The schematic of the whole model used in the simulation is presented in Fig. 2. In this study, the muscle activation and hence the muscle FES force or FES joint torque is varied through modulation of the PW ( $\left.P W_{\text {stim }}\right)$ of the stimulation signal $[8,17]$ keeping the stimulation frequency $f_{\text {stim }}$ fixed at $25 \mathrm{~Hz}$.

The leg model was equipped with the SBO model with optimized SBO spring parameters resulting from Huq and Tokhi [25], with the value of spring constant and spring natural angle as $3.0382 \mathrm{Nm}$ and $107.996^{\circ}$

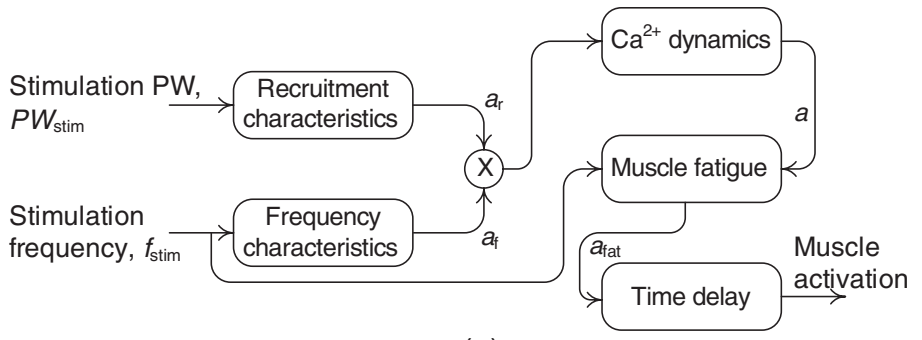

(a)

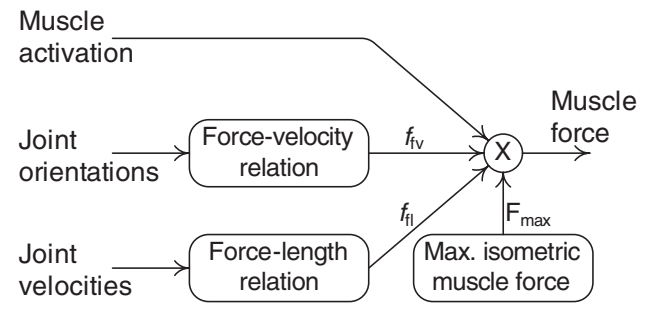

(b)

Fig. 1. Components of muscle dynamics (a) activation dynamics and (b) contraction dynamics [34]. 


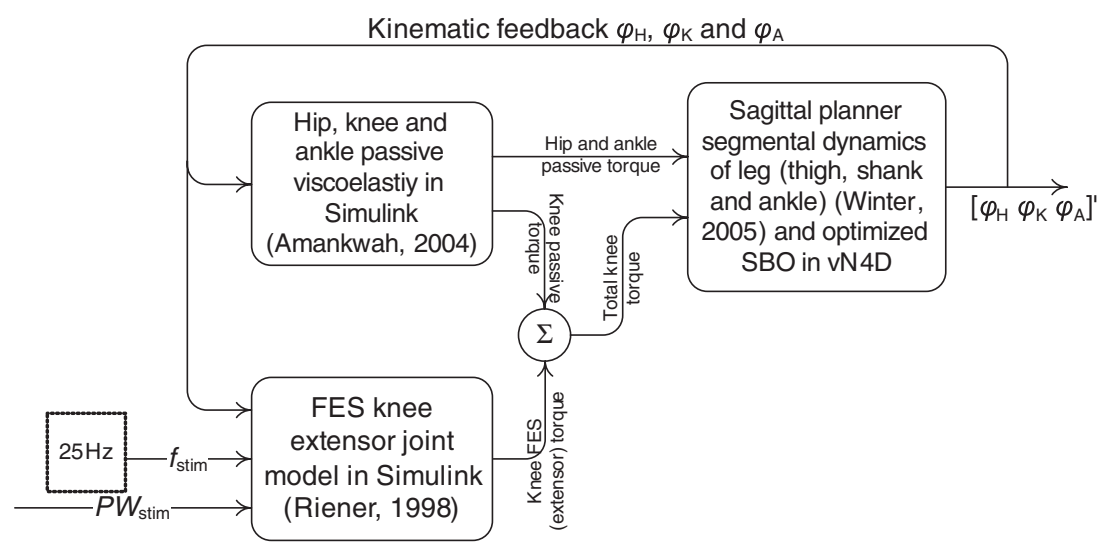

Fig. 2. The simulation model schematic. $\varphi_{\mathrm{H}}, \varphi_{\mathrm{K}}$ and $\varphi_{\mathrm{A}}$ are hip, knee and ankle angles respectively.

(corresponding to knee joint angle of $72.004^{\circ}$ ) respectively.

\subsection{Controllers explored}

In this paper, new control strategies suitable for the FES driven SBO, are investigated within simulation settings. The control strategies, amongst other features, include either PID or FLC as the basic controller blocks.

\subsubsection{PID control}

A PID controller is the most popular form of control loop feedback mechanism widely used in industrial control systems. A PID controller attempts to correct the error between a measured process variable and a desired set-point by calculating and then outputting a corrective action (control variable) that can adjust the process accordingly (Fig. 3). The value of the control variable depends on the three separate parameters; the proportional, the integral and derivative values.
The proportionalvalue determines the reaction to the current error, the integral value determines the reaction based on the sum of recent errors, and the derivative value determines the reaction based on the rate at which the error has been changing. The weighted sum of these three actions is used to adjust the process via a control element such as the position of a control valve or the power supply of a heating element, or for the current case, the stimulation intensity delivered to the paralysed muscle.

\subsubsection{Fuzzy logic control}

Like the ubiquitous PID controller, FLC on the other hand is now the fastest growing soft computing tool in the industry [19]. FLCs are rule-based systems that use fuzzy linguistic variables to model human ruleof-thumb approaches to problem solving, and thus overcome the limitation that classical expert systems may meet because of their inflexible representation of human decision making. The major strength of fuzzy

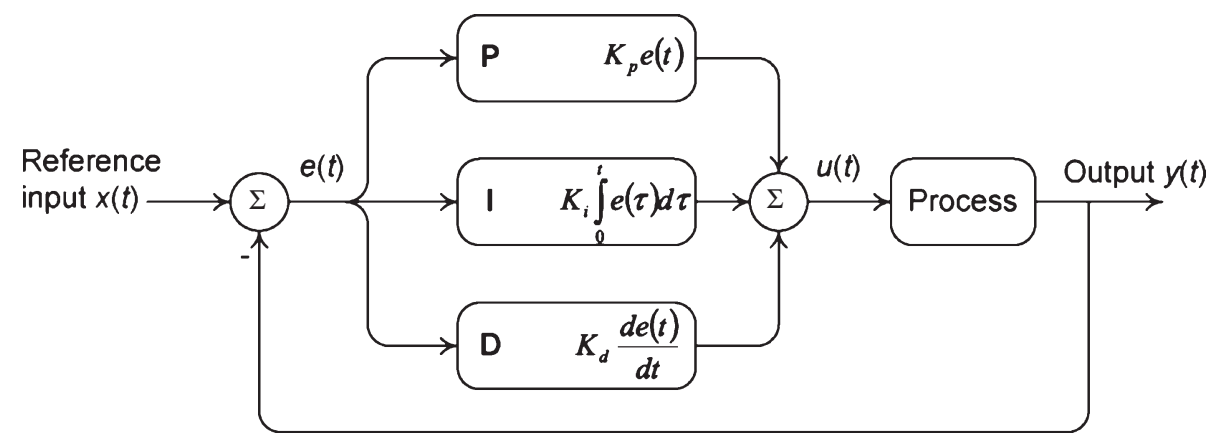

Fig. 3. PID controller schematic. 


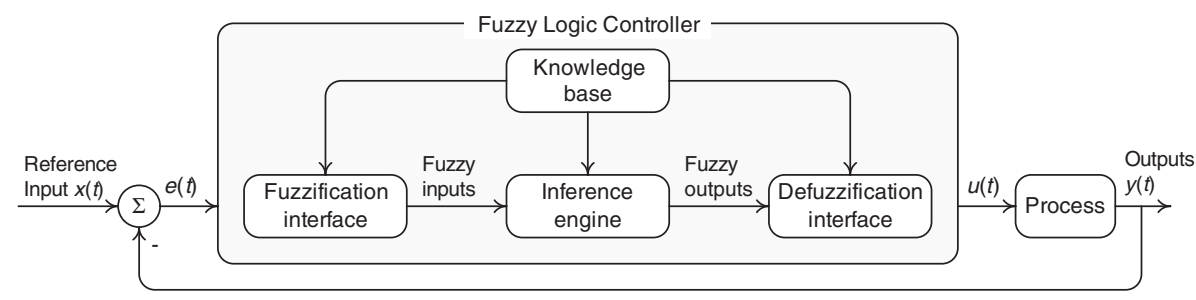

Fig. 4. Schematic of the FLC with basic structure.

controllers also lies in the way a nonlinear output mapping of a number of inputs can be specified easily using fuzzy linguistic variables and fuzzy rules [5].

A fuzzy control system in its basic form consists of four conceptual components: knowledge base, fuzzification interface, inference engine, and defuzzification interface [28, 29]. Figure 4 shows the block diagram of a fuzzy control system. The knowledge base contains all the controller knowledge and it comprises a fuzzy control rule base and a data base. The data base is the declarative part of the knowledge base which describes definition of objects (facts, terms, concepts) and definition of membership functions (MFs) used in the fuzzy control rules. The fuzzy control rule base is the procedural part of the knowledge base which contains information on how these objects can be used to infer new control actions. The inference engine is a reasoning mechanism which performs inference procedure upon the fuzzy control rules and given conditions to derive reasonable control actions. It is the central part of a fuzzy control system. The fuzzification interface (or fuzzifier) defines a mapping from a real-valued space to a fuzzy space, and the defuzzification interface (or defuzzifier) defines a mapping from a fuzzy space defined over an output universe of discourse to a real-valued space. The fuzzifier converts a crisp value to a fuzzy number while the defuzzifier converts the inferred fuzzy conclusion to a crisp value [32].

The principal constituents of the FLCs with reference to the current work are discussed below:

2.2.2.1. Fuzzy-state variable: In our simulation, the fuzzy state variable FLC consists of the error $e(t)$ (i.e. deviation of the actual output from its desired value) and its time derivative $\Delta e(t)$ as the input variable along with stimulation pulse-width (PW) $\left(P W_{\text {stim }}\right)$ as the control or output variable $u(t)$.

2.2.2.2. Fuzzification: The physical values of the input state variables are first normalized using scal-
Table 1

FLC rule base used in FES controllers

\begin{tabular}{llllll}
\hline Error & \multicolumn{5}{c}{ Derivative of error } \\
\cline { 2 - 6 } & NB & NS & ZO & PS & PB \\
\hline NB & NB & NB & NB & NS & ZO \\
NS & NB & NB & NS & ZO & PS \\
ZO & NB & NS & ZO & PS & PB \\
PS & NS & ZO & PS & PB & PB \\
PB & ZO & PS & PB & PB & PB \\
\hline
\end{tabular}

ing factors to map them into a normalized universe of discourse. Each universe of discourse associated with each state variable is divided into five fuzzy subsets: positive big (PB), positive small (PS), zero (ZO), negative small (NS), negative big (NB), with triangular MFs determining the degree of membership (Table 1).

2.2.2.3. Inference method: Table 1 summarizes the control rules or rule base for the controllers encompassing all possible AND combinations of the input fuzzy values. The inference method employs MAXMIN method where the output MF of each rule is given by minimum ( $\mathrm{min}$ ) operator and combined fuzzy output is given by maximum ( $\max$ ) operator.

\subsection{Controller tuning}

In case of a PID controller, the tuning operation applies to the three parameters, P, I and D. For the last half a century, numerous methods for tuning PID controllers have been developed. From the original works of Ziegler and Nichols [43] and Cohen and Coon [6], a great number of methods have been proposed [3, 42] including approaches to obtain PID optimal gains.

Being inherently knowledge based system; FLCs on the other hand have traditionally been tuned partly through experience and partly through trial-and-error. Besides, the lack of a mathematical description renders it unsuitable to tune through algorithms similar to the traditional tuning routine. 
Moreover, far away from a transfer function form, the overall model of the plant being considered is a multi-input-multi-output (MIMO) nonlinear model consisting of nonlinear lumped parameter model of the passive joint viscoelasticity and FES muscle (in Simulink) and nonlinear segmental dynamics (in vN4D). Given such plant model in the control loop, the conventional stability analysis and tuning techniques are of no use.

Consequently the tuning operations for both PID and FL controllers were formulated as optimization procedures using both GA [23] and MOGA [12]. Various carefully chosen objective functions were formulated with different control schemes, although the stability was not one of them because of the reasons discussed above. While in case of PID controllers there are only 3 decision variables, P, I and D; the FLCs were optimized involving 73 decision variables or parameters. Below is the list of parameters associated with FLCs that constituted the search space for the optimization procedure.

- 3 parameters associated with the scaling factors of the three fuzzy state vectors relating them to the normalized universe of discourse used by the inference method.

- 45 parameters defining a total of $15 \mathrm{MFs}$, with 10 input and 5 output MFs.

- 25 weighting factors including one for each rule.

\subsection{Control scheme-I}

Compromising with the natural dynamics of the plant in the control of movement to produce a desired outcome is not new. One such approach by Williamson [40] suggests to exploit portions of the dynamics that are particularly chosen for certain tasks resulting in a scheme which by working with rather than against the natural dynamics of the system, reaps the benefits of simplicity, robustness and efficiency. Perhaps the feature is most prominent within natural movements, performed by human or animals, as is suggested by the 'minimum torque-change' model of voluntary human arm movement by Uno et al. [37] and similar other ideas [11, 20, 31].

While the control scheme is closed-loop tracking control of the SBO knee joint, in a conventional manner, efforts are made to capture the dynamics of the SBO equipped leg into the chosen trajectory for the closed-loop FES control of the SBO. This scheme emphasises the choice of the reference trajectory with a view to overcome some drawbacks of the trajectory based closed-loop FES control, viz. poor tracking, oscillating response and inability to reach full knee extension angle [21]. Both PID and FL controllers are investigated in the scheme. Figure 5 shows the schematic of the control scheme.

\subsubsection{Reference trajectory derived from passive oscillation}

In this approach the reference trajectory for the knee joint is obtained by observing the segment's passive oscillation. Previous simulation work showed that the SBO equipped leg segment in its passive form (without FES control) behaves very much like a spring-mass system, exhibiting oscillation in its natural frequency, part of which basically constitutes earlier part of the SBO swing, until the FES is applied. With a view to make up the SBO swing phase of purely passive oscillation to its maximum extent, the reference trajectory in this case is drawn by stretching its own passive oscillation trajectory in both time and angle, retaining its original shape (see Fig. 6).

\subsubsection{Reference trajectory derived from cycle-to-cycle control}

FES induced movements have traditionally been achieved through application of stimulus bursts rather than continuous tracking control. The burst of stimulus signal would drive the joint to its desired orientation

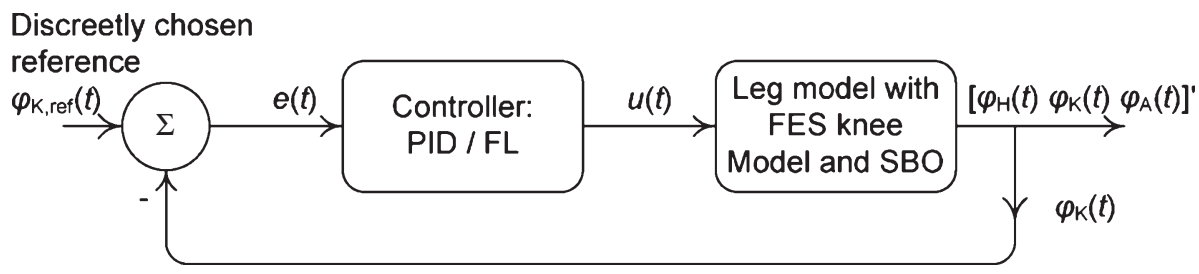

Fig. 5. SBO control scheme-I: closed-loop control of SBO with discreetly chosen trajectory. $\varphi_{\mathrm{H}}(t), \varphi_{\mathrm{K}}(t)$ and $\varphi_{\mathrm{A}}(t)$ are hip, knee and ankle joint trajectories respectively. 


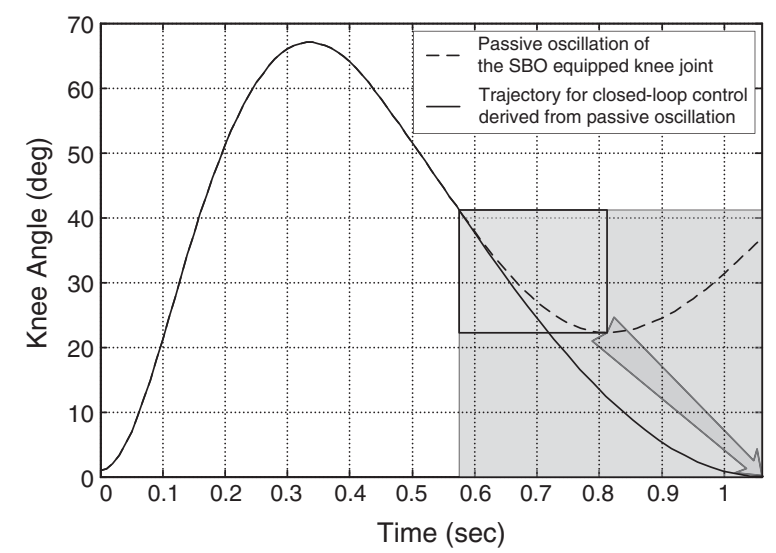

Fig. 6. Reference knee joint trajectory for SBO control derived from observing pure passive oscillation of the knee joint.

through ballistic movement and thus traversing a trajectory defined purely by the physics of the segment combination. The cycle-to-cycle control approach retains this basic mechanism of movement generation through stimulus burst and comes into action when the movement is repetitive or cyclical, through automatic adjustment of the burst parameters to maintain the desired target orientation at each cycle [13]. While the trajectory based closed-loop control for knee joint angle of paraplegic has been criticized for having poor tracking and oscillatory response and even its inability to reach full knee extension angle [21], the ability of the cycle-to-cycle control approach to realize the target joint orientation has been demonstrated in experimental tests of controlling maximum knee extension angle [38] or hip joint range [13].

To find out the trajectory traversed by the knee joint when driven by a suitably parameterized stimulus burst to full extension, the temporal parameters of the burst of stimulus signal with $250 \mu \mathrm{sec}$ PW and stimulation frequency of $25 \mathrm{~Hz}$ to be applied at the knee extensor of the leg model is optimized. In this case, the parameters to be optimized include the position of the burst (burststart) in time scale and its duration (burst-width) (see
Fig. 7). As the optimized burst is expected to be just enough to drive the knee joint to full extension, the primary objective is to minimize the magnitude of the overshoot or undershoot (negative overshoot) from the desired target knee orientation $\left(0^{\circ}\right.$ for the current case):

$$
\mathrm{f}_{1}(y)=(\text { overshoot })^{2}
$$

Since it is possible that there are multiple solutions meeting the primary objective; to be able to select the best solution amongst them, a second objective function $\mathrm{f}_{2}(y)$ is defined as the time-integral of the active torque from the knee extensor, a criterion related to the energy consumption of the muscle [9]:

$$
\mathrm{f}_{2}(y)=\int_{0}^{t} M_{\text {act }}(t) \mathrm{dt}
$$

where $M_{\text {act }}(t)$ is the active FES torque from the stimulated muscle.

As a whole, the optimization procedure is supposed to find the parameter of the stimulus burst that would drive the SBO knee to its full extension, but with least amount of energy consumption by the muscle.

\subsection{Control scheme-II}

In principle, this control approach is similar to the discrete-time cycle-to-cycle control which appreciates the importance of reaching a target joint orientation through ballistic movement, rather than through rigorously following a predefined trajectory $[13,38]$. But unlike cycle-to-cycle control, the target joint orientation is achieved through part-time closed-loop control with constant value of the target joint orientation as the set-point. Besides its suitability in generating the target joint orientation, one of the attractions of the cycle-tocycle control in FES application is the absence of any reference trajectory. The current SBO control scheme (control scheme-II) shares this feature with the cycleto-cycle control and does not employ the so called reference trajectory. Yet it is still closed-loop control, but only during part of the swing phase with simply the target joint orientation as the set-point. In effect

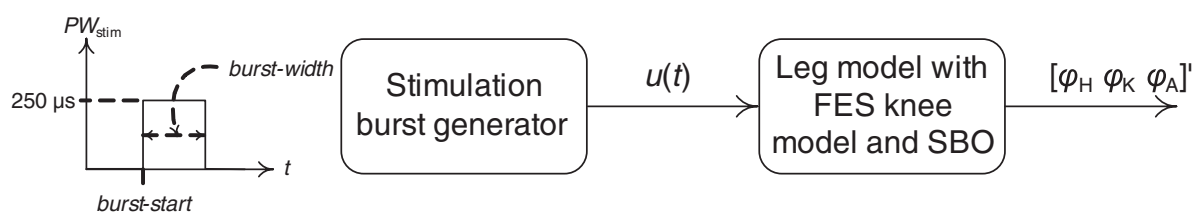

Fig. 7. Schematic of cycle-to-cycle control structure initialized/optimized with GA. 


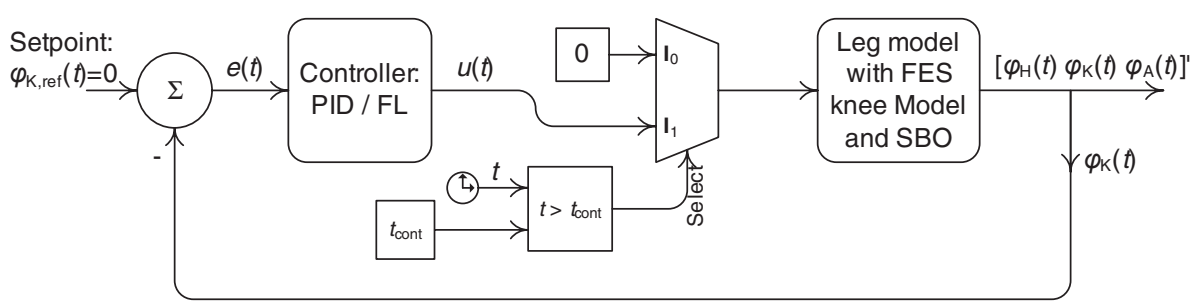

Fig. 8. Schematic of SBO control scheme-II: part-time closed-loop control with constant set-point.

the scheme can be seen as closed-loop control with a step input but only for a duration required to produce the full knee extension. The approach is shown schematically in Fig. 8. As can be seen, the set-point to the controller is the target joint orientation and the controller is only effective after certain time period $t=t_{\text {cont }}$. During the period $t=0$ till $t=t_{\text {cont }}$, the leg segment is left to oscillate entirely passively.

The control scheme is tested with both PID and FL controllers. As in control scheme-I, 3 parameters for the PID controller and 73 parameters associated with the FLC are optimized using the same MOGA routine for objectives to be discussed below. In addition to the controller parameters, the new parameter unique to the control scheme, the time $t_{\text {cont }}$, which marks the onset of the control operation is also optimized using the MOGA.

Considering that the control approach must be able to produce full knee extension without any overshoot or undershoot (lack of extension), the first and foremost objective function to be minimized is the magnitude or absolute value of the overshoot. Thus, one of the objectives is defined as in equation (1). Apart from reaching the target orientation, in FES applications it is also important to take measures relating to possible minimization of the muscle fatigue. Hence, a second objective function, which is related to the energy consumption by the muscle, and thus the resultant fatigue, is defined as the time-integral of the FES induced active torque $M_{\text {act }}, f_{2}(y)$ in equation (2).

\section{Results}

\subsection{Control scheme - I with reference trajectory derived from passive oscillation}

Both PID and FL controllers are tested to perform this control operation. Following the work of
Grefenstette [18], optimization of the PID controller is done using a GA routine which is initialized as follows:

$\begin{array}{ll}\text { Number of individuals } & 50 \\ \text { Number of generations } & 100 \\ \text { Crossover rate } & 80 \% \\ \text { Mutation rate } & 1 \%\end{array}$

The controller optimization process would normally include the time-integral of the absolute value of the error between the reference and actual output, and the objective function for the controller optimization would normally be the time-integral of the absolute or squared value of the error between the reference and actual trajectory. But in practice, the most important objective of such FES control would be to attain full knee extension regardless of its tracking accuracy, and hence should be considered in the optimization process while choosing the objective function. Timeweighting the error between the reference and actual trajectory in evaluating the objective function could be an option, as it would make the error more and more prominent as the full extension of the trajectory is approached and influence the optimization process accordingly. The objective function to be minimized was thus defined as the integral of the time-weighted squared error (ITSE):

$$
\mathrm{f}(y)=\int_{0}^{t}\left(y_{\mathrm{ref}}(t)-y_{\mathrm{act}}(t)\right)^{2} t \mathrm{dt}
$$

where $y_{\text {ref }}(t)$ and $y_{\text {act }}(t)$ are the reference- and actual knee joint trajectories respectively.

Figure 9 shows the actual knee joint trajectories with the optimized controllers. Even after running the optimization for 100 generations, both the controllers seem to have failed to drive the knee to full extension. This obviously makes the control scheme futile as achieving full knee extension cannot be compromised 


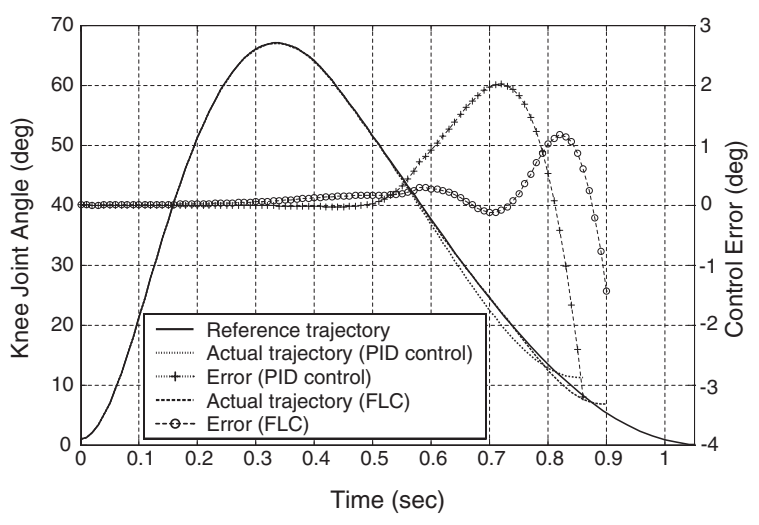

Fig. 9. FES control of SBO with reference trajectory derived from passive oscillation.

in assistive gait generation. Since failure occurred even with utmost precautions in place through the choice of controller, controller optimization and choice of objective function; the direction of investigation is slightly steered away from just tuning the controllers: finding a suitable reference trajectory.

As suggested in the originally proposed SBO concept, the hip flexion (achieved entirely passively as a result of the dynamic coupling to the ipsilateral knee flexion) is held by the ratchet mechanism at its flexion peak. Therefore, the amount of hip flexion thus maintained is actually determined by the knee flexion peak and hence the SBO spring parameters. It is therefore independent of the FES control which normally applies following the hip lock and is the same as was in part-I of this work [25] in all cases. Thus, it is deemed redundant to make reference to hip trajectory in the current work.

\subsection{Control scheme - I with reference trajectory derived from cycle-to-cycle control}

This control scheme is discussed in Section 2.4.2. In order to derive the trajectory that would be traversed by the knee joint in case of cycle-to-cycle control, a MOGA with 50 binary coded individuals was run for 100 generations to optimize the burst parameters (Fig. 7) simultaneously minimizing both $\mathrm{f}_{1}(y)$ and $\mathrm{f}_{2}(y)$ (equations (1) and (2) respectively). The crossover and mutation rate were set to be the same as in the last GA routine ( $80 \%$ and $1 \%$ respectively). The resulting nondominated solution front after 100 generations along with the table containing the corresponding parameter values is shown in Fig. 10. The best solution in terms of $\mathrm{f}_{1}(y)$, which would provide just enough torque to produce full knee extension is highlighted in the figure.

Figure 11 shows the stimulation burst, the active torque it generates and above all, the resultant knee joint trajectory corresponding to the highlighted solution in Fig. 10. The result just reaffirms the superiority of the cycle-to-cycle control in achieving target joint orientation.

Since the cycle-to-cycle control approach, unlike closed-loop tracking control, can produce full knee extension and does not impose any predefined trajectory for the SBO knee, the course of trajectory it results is evidently a feasible trajectory which must have been defined by the total dynamics of the overall model

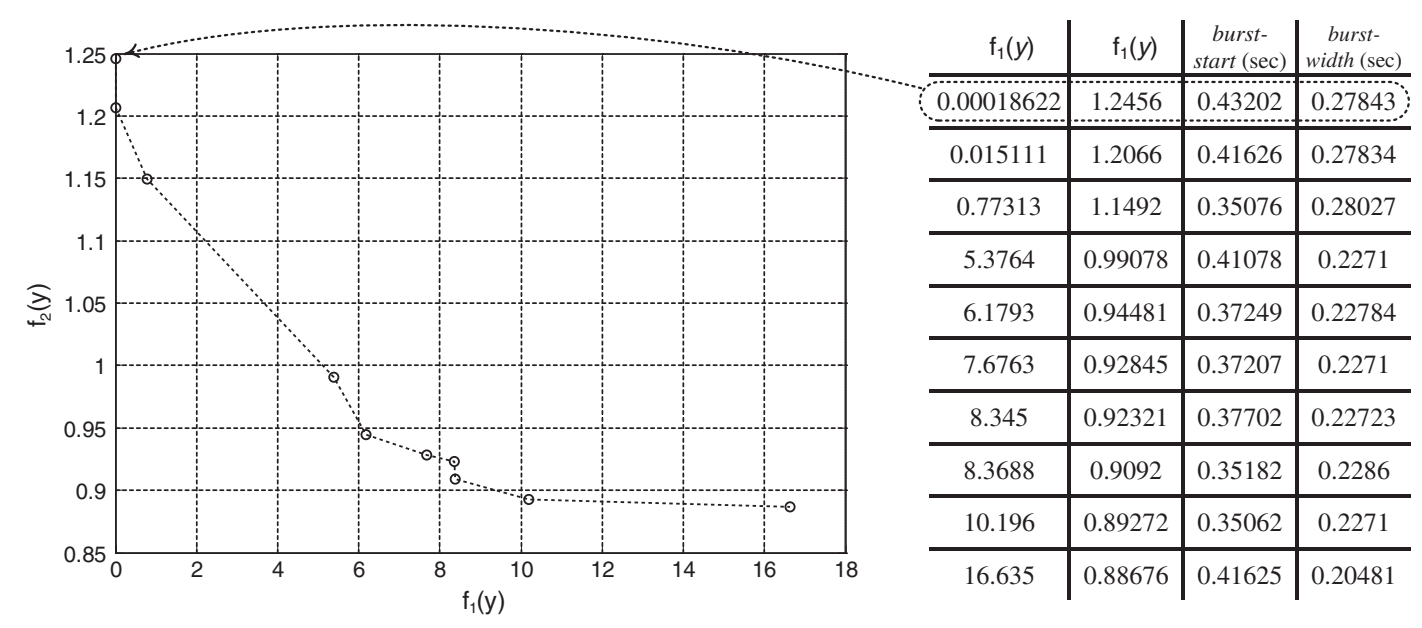

Fig. 10. MOGA optimization/initialization of cycle-to-cycle control: non-dominated solution front (left) and list of optimal parameters (right). 


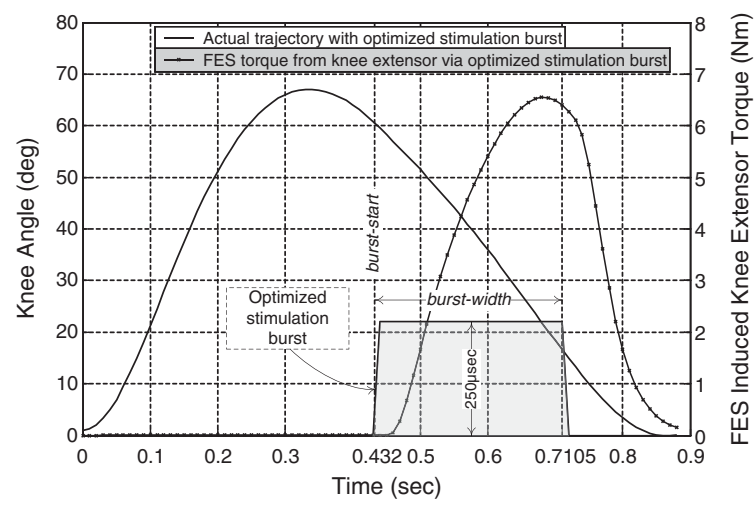

Fig. 11. MOGA optimized cycle-to-cycle control: stimulus burst, FES induced active torque and knee joint trajectory.

being considered (leg segment, FES and viscoelastic joint model and SBO). In other words it may be said that, the trajectory was not chosen for the leg model, rather the leg model itself chose the trajectory for it to be able to achieve full knee extension. Recognising the uniqueness of the resultant trajectory, it is hypothesised that it might constitute a more suitable reference trajectory to be used in the closed-loop SBO control and is used in the following control operation.

As before, both PID and FL controllers are tested in this scheme in closed-loop configuration but with the reference trajectory derived in Fig. 11. Optimization of the PID controller is performed with the same GA routine as before with the same ITSE criterion defined as the objective function, equation (3).

In case of the FLC, unlike before where the only objective function was the ITSE (equation 3), an additional objective function; the sum of the weighting factors of the fuzzy rules, is considered:

$$
\mathrm{f}_{3}(y)=\sum_{i=1}^{n} w_{i}
$$

where, $w_{\mathrm{i}}$ is the $i$ 'th rule with $n$ represents the total number of rules. The idea behind choosing this objective function is to identify the redundant rules as the optimization process will achieve this through minimizing each of them individually. The same MOGA routine used in the stimulus burst optimization is used in this case, with the newly defined objective functions.

Figure 12 shows the results related to the optimization process of both the controllers. Figure 12a shows the convergence of the objective function ITSE with generation for the PID controller tuning, which can be seen to stop converging any more after 60 generations. Also plotted in Fig. 12a are the best values of the same objective (ITSE); $\mathrm{f}_{1}(y)$ for the FLC optimization at certain intervals of generations during the MOGA process. With a lower value for the FLC, this clearly exhibits superior performance of the FLC in terms of ITSE almost throughout 100 generations. Figure $12 \mathrm{~b}$ on the other hand shows the non-dominated solution fronts after 20, 40, 60, 80 and 100 generations of the FLC optimization using MOGA. The solution fronts also exhibit convergence as they move towards lower values for both the objectives with generation.

The optimized values of the $\mathrm{P}$, I and $\mathrm{D}$ terms for the PID controller were found as $0.11963,0.779$ and 1.5187. For the optimized FLC (with lowest value of ITSE or $\left.f_{1}(y)\right)$ the scaling factors for the

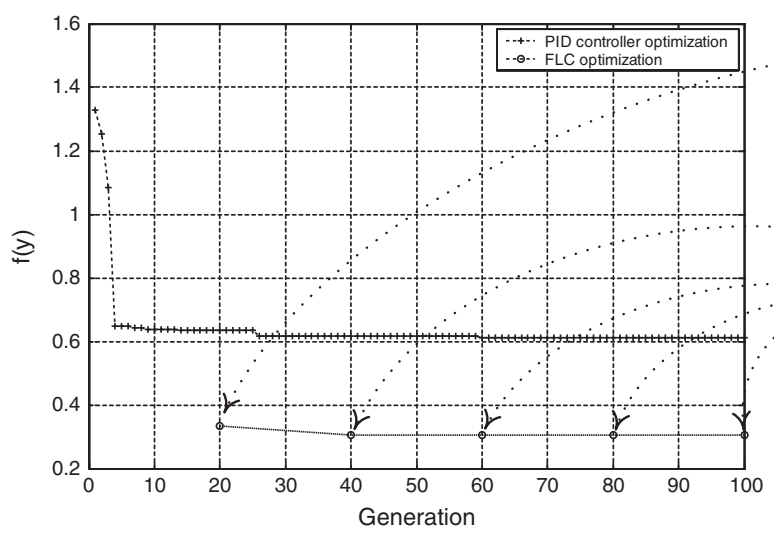

(a)

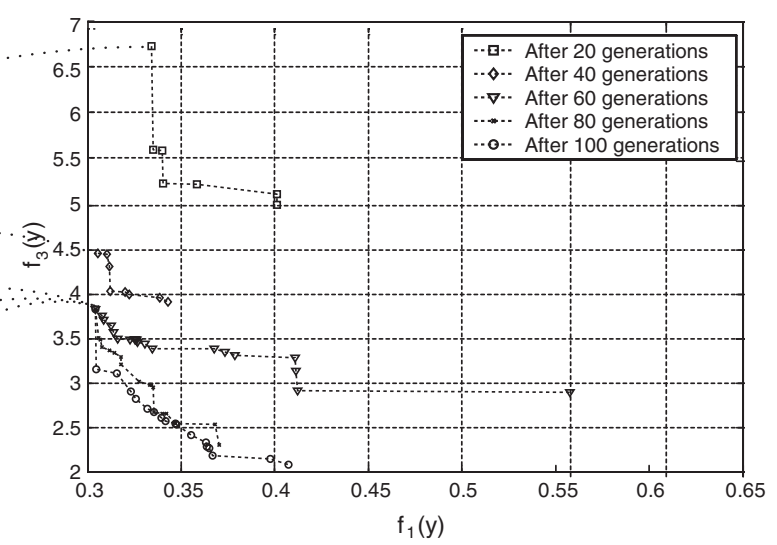

(b)

Fig. 12. (a) Convergence of objective functions with generation for both PID and FL controller optimization, (b) Non-dominated solution fronts for MOGA optimization of FLC. 


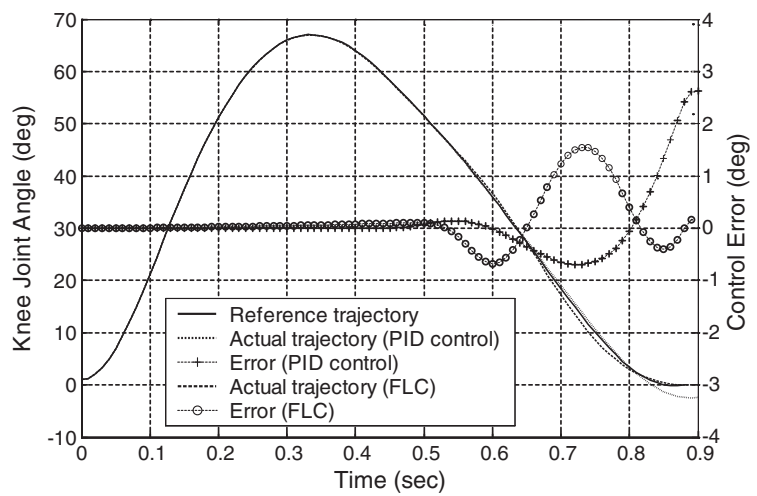

Fig. 13. Reference and actual trajectory and tracking error with PID and FL control scheme-I.

proportional- and derivative input and for the output were $0.42132,0.74246$ and 680.94 respectively.

Following optimization of the FLC, the rules weighing less than 0.2 are deemed insignificant and are highlighted by the shaded cells. These rules are ignored in the final controller, significantly reducing the number of rules from 25 to 8 .

Figure 13 shows the knee joint trajectories obtained using the optimized PID and FL controllers along with the reference trajectory, which is drawn from the cycleto-cycle control. The first and foremost striking aspect of this result is how both controllers in their closed-loop configuration were able to generate full knee extension given this particular trajectory. This in turn strengthens our hypothesis about the characteristic trajectory that it is supposed to be more suitable as reference trajectory than trajectory derived through some other considerations for closed-loop operation. The FLC was found to produce better outcome without any overshoot whereas the PID controller produced some overshoot, rendering its error higher than that with FLC.

Figure 14 gives a clearer insight into the operations of the controllers through comparison between the outputs from the controllers and active torque generated during the control. Despite its slightly inferior performance in input tracking, the PID controller seems to have accomplished the task with much lower as well as smoother (with no switching) control output $\left(P W_{\text {stim }}\right)$ than the FLC, which can ultimately be expected to contribute to lower muscle fatigue. On the contrary, the control output from the FLC appears to possess a high magnitude, switching and above all, the steady-state error found in conventional PD controllers. Thus, the results reveal the superior performance of the FLC in

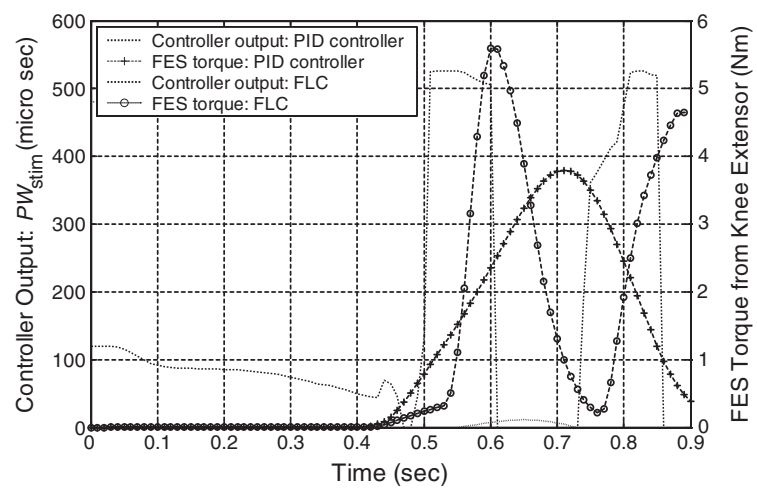

Fig. 14. Controller output and resultant FES induced active torque from PID and FL controller (control scheme-I).

terms of input tracking, but they also reveal the cost it comes at for FES applications.

\subsection{Control scheme - II: Part-time closed-loop control with target orientation as set-point}

The control scheme is tested with both PID and FL controllers. As in control scheme-I, 3 parameters for the PID controller and 73 parameters associated with the FLC are optimized using the same MOGA routine for objectives to be discussed below. In addition to the controller parameters, the new parameter unique to the control scheme, the time $t_{\text {cont }}$, which marks the onset of the control operation is also optimized using the MOGA.

Considering that the control approach must be able to produce full knee extension without any overshoot or undershoot (lack of extension), the first and foremost objective function to be minimized is the magnitude or absolute value of the overshoot. Thus, one of the objectives is defined as in equation (1). Apart from reaching the target orientation, in FES applications it is also important to take measures relating to possible minimization of the muscle fatigue. Hence, a second objective function, which is related to the energy consumption by the muscle, and thus the resultant fatigue, is defined as the time-integral of the FES induced active torque $M_{\text {act }}, f_{2}(y)$ in equation (2).

The previously used MOGA routine is then used to tune both the controllers and their associated $t_{\text {cont }}$ through simultaneously minimizing both objectives $\mathrm{f}_{1}(y)$ and $\mathrm{f}_{2}(y)$. Figure 15 shows the Pareto-optimal solution front for both the controllers indicating better 


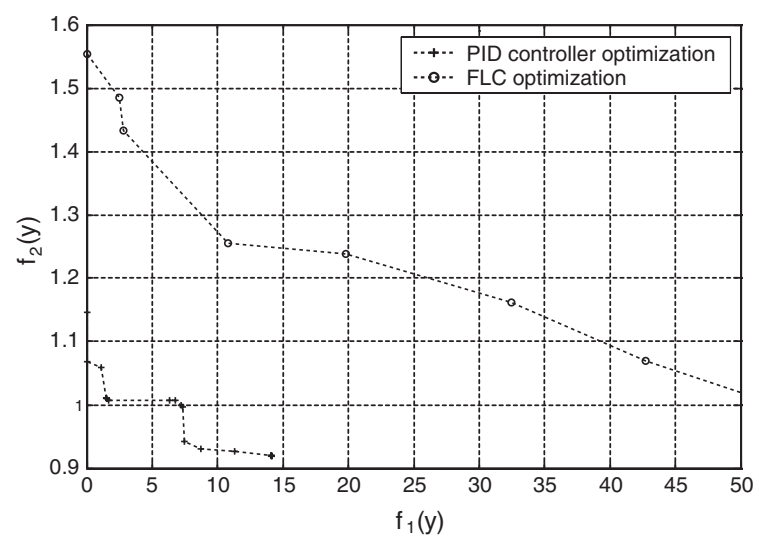

Fig. 15. Non-dominated solution front for MOGA optimization of PID and FL controller under control scheme-II.

performance (convergence) by the PID controller than the FLC.

Since reaching full extension is the main priority, the parameters corresponding to the non-dominated solution with minimum value for the $\mathrm{f}_{1}(y)$ are chosen. For the PID controller, the optimum value (in terms of $\mathrm{f}_{1}(y)$ ) for the P, I and D terms were found to be 5.15, 0.67711 and 4.1339 respectively, whereas for the FLC (with lowest value of ITSE or $\mathrm{f}_{1}(y)$ ) the scaling factors for the proportional- and derivative input and for the output were $0.50068,0.62453$ and 641.27 respectively. The optimal values for the newly introduced parameter, $t_{\text {cont }}$ for the PID and FL controller were found to be $0.4056 \mathrm{sec}$ and $0.4849 \mathrm{sec}$ respectively.

Figure 16 shows the knee joint trajectories obtained using the optimized controllers. As can be seen, both the controllers were able to drive the knee to its full extension through a smooth trajectory with the FLC producing slightly larger potential swing duration.

The controller output $u(t)$ modulating the $P W_{\text {stim }}$ and the resultant FES induced muscle torque $M_{\text {act }}$ are shown in Fig. 17 for both controllers. The controller output for both the controllers are like spikes appearing at $t=t_{\text {cont }}$, when the controllers actually come into play.

\subsection{Fatigue resistance of the controllers}

The fatigue resistance of all the SBO control approaches considered are analysed based on 3 criteria:

- criterion (a): Time-integral of the control variable $u(t)$, or the stimulation intensity $P W_{\text {stim }}$.

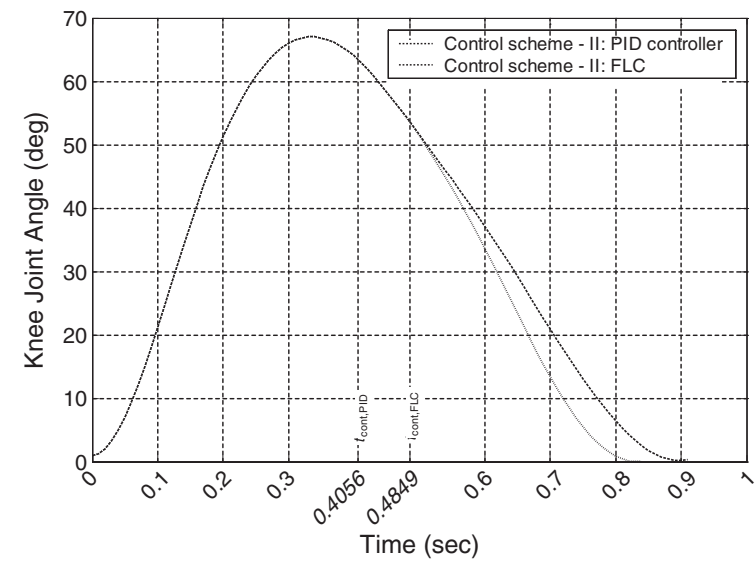

Fig. 16. Knee joint trajectories using PID and FL controller under control scheme-II.

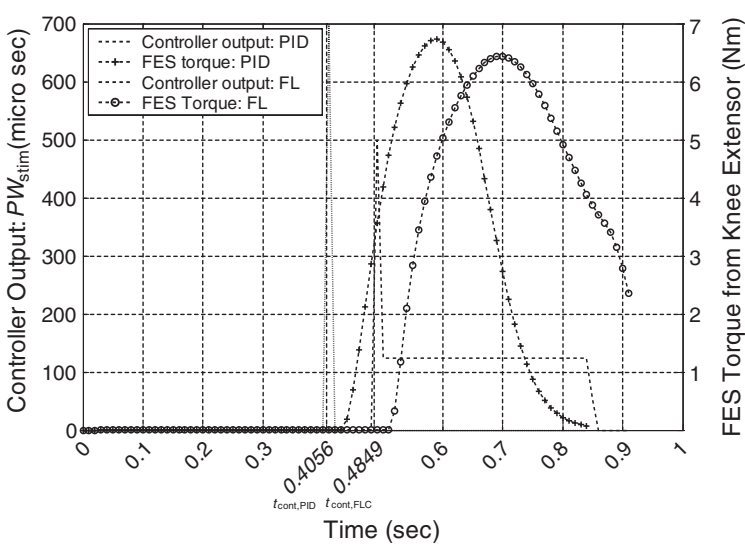

Fig. 17. Controller output and resultant FES induced active torque from PID and FL controller (control scheme-II).

- criterion (b): FES induced mechanical energy output from the muscle.

- criterion (c): Relative drop in knee extension with reduced (fatigued) muscle torque output.

The first two criteria are directly related to the amount of fatigue generated within the muscle and emanate from relation of the muscle fatigue to the energy consumption in the muscle which in turn depends on the time-integral of activation of the muscle $\left(P W_{\text {stim }}\right.$ in this study) [39]. The third criterion on the other hand reflects the robustness of the particular control approach.

The results are shown in Figs. 18-20. Figure 20 is the final part of the knee joint trajectory obtained using the same controllers, but with FES torque dropped down to $80 \%$ of its normal value. Table 2 and the bar 


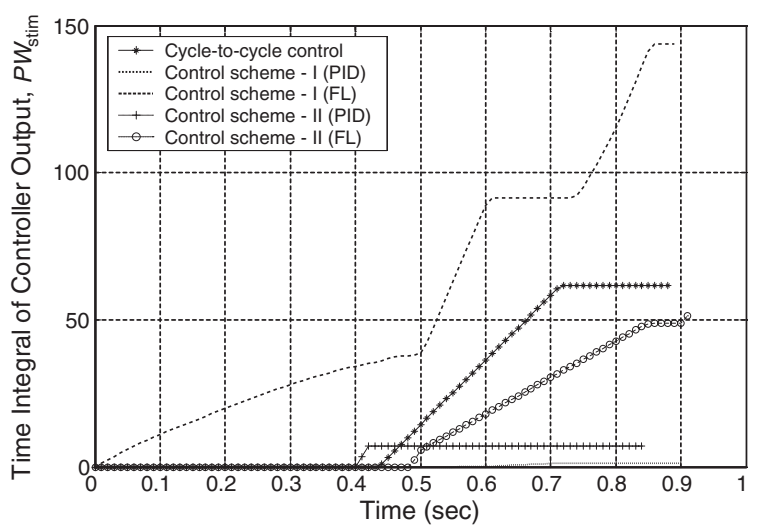

Fig. 18. Time-integral of the controller output $u(t)$ modulating $P W_{\text {stim }}$ for the control approaches considered in this study.

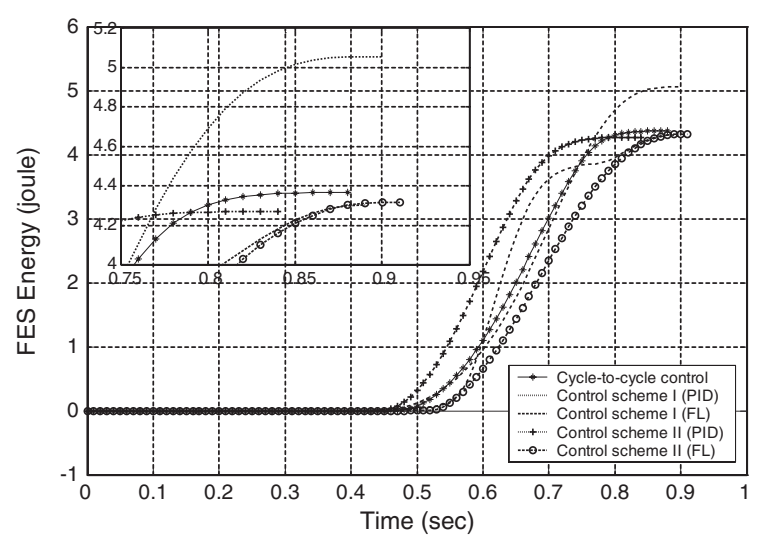

Fig. 19. FES induced mechanical energy input into plant for different control approaches. Magnified end values in the inset.

graph of Fig. 21 summarise the results based on these 3 criteria. Especially the bar graph portrays a clear picture of these criteria associated with the control approaches. As the lower value of a criterion the better fatigue resistant the control approach is, the result seems to rank different control approaches differently under different criteria and thus makes it difficult to

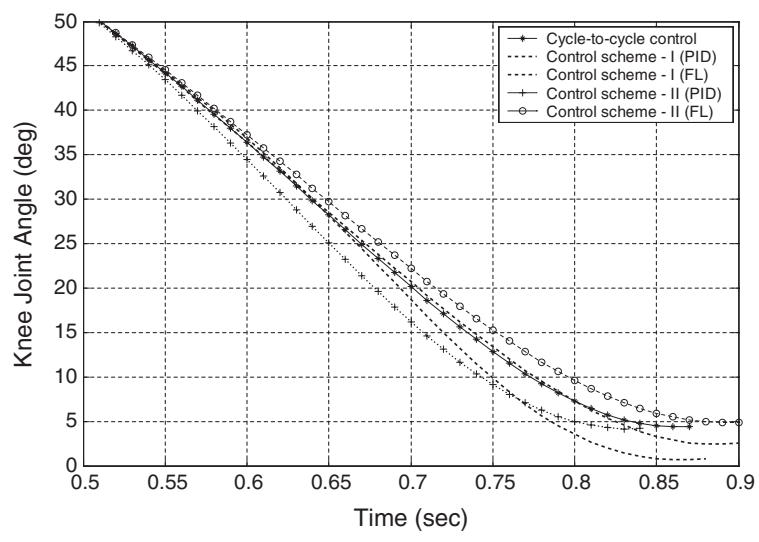

Fig. 20. Knee extension generated with FES muscle force output $80 \%$ of its normal value (simulated fatigue).

draw conclusive comparison of their fatigue resistance capability.

The most conspicuous of all is the extraordinary performance by the PID controller both in scheme-I and II with regard to criterion (a), whereas it does not perform quite well (comparatively) in terms of the other two criteria. Another feature worth noting is the robustness of the FLC under scheme-II as the knee extension drops by $1.129 \%$ with FES torque of $80 \%$ of its normal value (criterion (c)). Above all, the newly proposed control schemes I and II can conclusively be commended as well performed compared to the traditional cycle-to-cycle approach.

\section{Discussion}

FES induced movement control is a challenging task. The inclusion of energy storage element on top of the segmental dynamics perhaps worsens the challenge. As the knee extensor is one of the strongest muscles in the human body, one might superficially anticipate it to be a simple task to generate full knee extension in a sprung knee through its stimulation as in

Table 2

Values of the 3 criteria defined to compare the fatigue resistance of the controllers

\begin{tabular}{lccc}
\hline Control & $\Sigma P W_{\text {stim }}$ & $\begin{array}{c}\text { FES energy input } \\
(\text { Joul })\end{array}$ & $\begin{array}{c}\% \text { fall in knee } \\
\text { extension }\end{array}$ \\
\hline Cycle-to-cycle & 61.6 & 4.3669 & 6.5471 \\
Scheme-I (PID) & 1.2503 & 5.0544 & 7.0063 \\
Scheme-I (FL) & 143.88 & 4.3034 & 1.1291 \\
Scheme-II (PID) & 7.1901 & 4.2716 & 6.1461 \\
Scheme-II (FL) & 51.344 & 4.3167 & 6.8104 \\
\hline
\end{tabular}



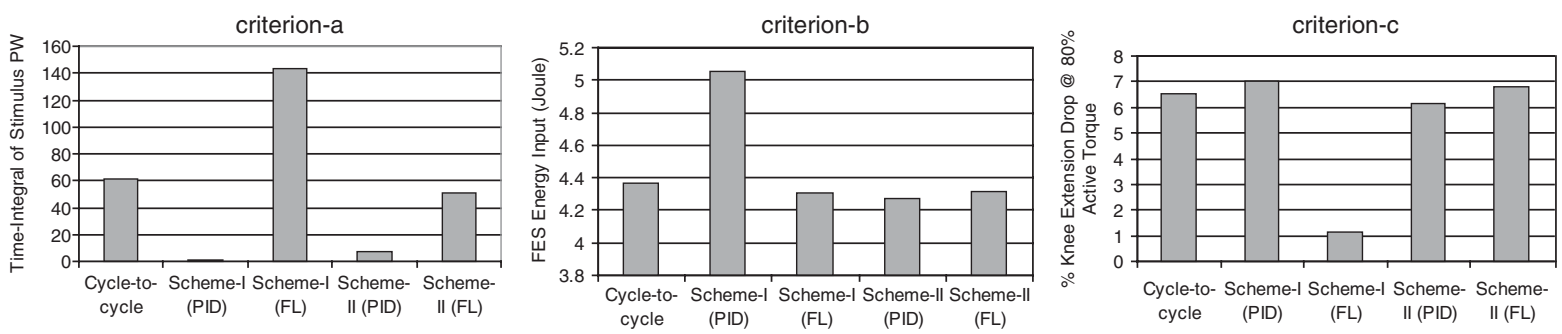

Fig. 21. Fatigue resistance of the SBO control approaches compared based on 3 criteria.

the case of SBO. But unfortunately this does not happen to be the case, not because of lack of torque from the muscle, rather due to the nonlinearity associated with it. In simple terms, even a strong muscle may not be capable of delivering adequate amount of torque at a time when it is required, given the orientations of the relevant joints at that instant.

Even with the advent of many sophisticated closedloop control algorithms, the open-loop pattern based control still reigns the field of practical solutions of FES induced movement. The control approaches proposed in this paper have been based on the appreciation of this fact and simultaneously blending it to closedloop strategy to retain the advantages of both the approaches to combine them into one. In principle, such approach can be a preferable form of control technique for any cyclical movement control. Especially in FES applications, due to the highly time-variant nature of the plant, any control algorithm must be accompanied by adaptive mechanisms for the controller parameters to compensate for the plant variations and the proposals in this paper are no exception.

Also due to the black-box nature of the model as well as the FLC within the control loop, it is beyond the scope of this work to address the issue of closed-loop stability, which is an essential element to be considered. This obviously demands further exploration. One strong candidate to resolve this issue associated with adaptation, nonlinearity and above all stability, is the fuzzy adaptive sliding mode control in which the sliding mode control techniques are utilized in the control design and adaptive fuzzy systems are employed to approximate the unknown system nonlinearities [27].

\section{Conclusions}

Two control schemes have been presented in this study for FES assisted movement control with special reference to application in SBO. The primary focus of these schemes is to minimize fatigue in FES muscle; a well known pitfall in FES applications. These schemes are novel in the sense that they prioritize the natural dynamics of the plant in the control operation. The proposed schemes have been shown to be able to successfully drive the limb joint to its required peak even with an apparent stringent demand on the actuator. GAs has been used to tune the controller for desired performances; especially to cause least amount of fatigue in the FES muscle. Such approach can extend the success of FES induced movement control much further as it addresses the primary issues restricting the real usability of FES.

\section{References}

[1] J.J. Abbas and H.J. Chizeck, Neural network control of functional neuromuscular stimulation systems: Computer simulation studies, IEEE Trans Biomed Eng 42(11) (1995), 1117-1127.

[2] K. Amankwah, R.J. Triolo and R. Kirsch, Effects of spinal cord injury on lower-limb passive joint moments revealed through a nonlinear viscoelastic model, J Rehabil Res Dev 41(1) (2004), 15-32.

[3] K.J. Astrom and T. Hagglund, Automatic tuning of simple regulators with specifications on phase and amplitude margins, Automatica 20(5) (1984), 645-651.

[4] H.B. Boom, A.J. Mulder and P.H. Veltink, Fatigue during functional neuromuscular stimulation, Prog Brain Res 97 409-418.

[5] T.C. Chin and X.M. Qi, Genetic algorithms for learning the rule base of fuzzy logic controller, Fuzzy Sets and Systems 97 (1998), 1.

[6] G.H. Cohen and G.A. Coon, Theoretical consideration of retarded control, Trans ASME 75(1) (1953), 827-834.

[7] P.E. Crago, N. Lan, P.H. Veltink, J.J. Abbas and C. Kantor, New control strategies for neuroprosthetic systems, J Rehabil Res Dev 33(2) (1996), 158-172.

[8] P.E. Crago, P.H. Peckham, J.T. Mortimer and J.P. Van der Meulen, The choice of pulse duration for chronic electrical stimulation via surface, nerve, and intramuscular electrodes, Ann Biomed Eng 2(3) (1974), 252-264. 
[9] R.D. Crowninshield and R.A. Brand, A physiologically based criterion of muscle force prediction in locomotion, J Biomech 14(11) (1981), 793-801.

[10] M. Ferrarin, F. Palazzo, R. Riener and J. Quintern, Modelbased control of FES-induced single joint movements, IEEE Trans Neural Syst Rehabil Eng 9(3) (2001), 245-257.

[11] T. Flash and N. Hogan, The coordination of arm movements - an experimentally confirmed mathematical-model, Journal of Neuroscience 5(7) (1985), 1688-1703.

[12] C.M. Fonseca and P.J. Fleming, Genetic algorithms for multiobjective optimisation: Formulation, discussion and generalization. Fifth International Conference on Genetic Algorithms, Morgan Kaufmann, San Mateo, CA, 1993.

[13] H.M. Franken, P.H. Veltink, G. Baardman, R.A. Redmeyer and H.B. Boom, Cycle-to-cycle control of swing phase of paraplegic gait induced by surface electrical stimulation, Med Biol Eng Comput 33(3 Spec No) (1995), 440-451.

[14] H.M. Franken, P.H. Veltink, M. Fidder and H.B.K. Boom, Fatigue of intermittently stimulated paralyzed human quadriceps during imposed cyclical lower leg movements, Journal of Electromyography and Kinesiology 3(1) (1993), 3-12.

[15] C.S. Gharooni, B. Heller and M.O. Tokhi, A new hybrid spring brake orthosis for controlling hip and knee flexion in the swing phase, IEEE Trans Neural Syst Rehabil Eng 9(1) (2001), 106-107.

[16] H. Gollee, K.J. Hunt and D.E. Wood, New results in feedback control of unsupported standing in paraplegia, Ieee Transactions on Neural Systems and Rehabilitation Engineering 12 (2004), 73-80.

[17] P.H. Gorman and J.T. Mortimer, The effect of stimulus parameters on the recruitment characteristics of direct nerve stimulation, IEEE Trans Biomed Eng 30(7) (1983), 407-414.

[18] J.J. Grefenstette, Optimization of control parameters for genetic algorithms, IEEE Transactions on Systems Man and Cybernetics 16(1) (1986), 122-128.

[19] A.D. Grey, Power Factor improvement using fuzzy logic control of an AC synchronous motor, IEEE Southeastcon, Ft. Lauderdale, FL, IEEE, 2005.

[20] Z. Hasan, Optimized movement trajectories and joint stiffness in unperturbed, inertially loaded movements, Biological Cybernetics 53(6) (1986), 373-382.

[21] M.S. Hatwell, B.J. Oderkerk, C.A. Sacher and G.F. Inbar, The development of a model reference adaptive controller to controlthe knee joint of paraplegics, IEEE Transactions on Automatic Control 36(6) (1991), 683-691.

[22] W. Holderbaum, K.J. Hunt and H. Gollee, H-infinity robust control design for unsupported paraplegic standing: Experimental evaluation, Control Engineering Practice 10(11) (2002), 1211-1222.

[23] J.H. Holland, Adaptation in Natural and Artificial Systems, The University of Michigan Press, Ann Arbor, 1975.

[24] K.J. Hunt, R.P. Jaime and H. Gollee, Robust control of electrically-stimulated muscle using polynomial $\mathrm{H}$ design, Control Engineering Practice 9(3) (2001), 313-328.

[25] M.S. Huq and O. Tokhi, Genetic algorithms based approach for designing spring brake orthosis - Part I: Spring parameters,
Applied Bionics and Biomechanics, (accepted for publication), 2012.

[26] R.P. Jaime, Z. Matjacic and K.J. Hunt, Paraplegic standing supported by FES-controlled ankle stiffness, IEEE Trans Neural Syst Rehabil Eng 10(4) (2002), 239-248.

[27] S. Jezernik, R.G. Wassink and T. Keller, Sliding mode closedloop control of FES: Controlling the shank movement, IEEE Trans Biomed Eng 51(2) (2004), 263-272.

[28] C.C. Lee, Fuzzy-Logic in Control-Systems - Fuzzy-Logic Controller - Part I, IEEE Transactions on Systems Man and Cybernetics 20(2) (1990a), 404-418.

[29] C.C. Lee, Fuzzy-Logic in Control-Systems - Fuzzy-Logic Controller - Part II, IEEE Transactions on Systems Man and Cybernetics 20(2) (1990b), 419-435.

[30] Z. Matjacic, K. Hunt, H. Gollee and T. Sinkjaer, Control of posture with FES systems, Med Eng Phys 25(1) (2003), 51-62.

[31] W.L. Nelson, Physical principles for economies of skilled movements, Biological Cybernetics 46(2) (1983), 135-147.

[32] K.M. Passino and S. Yurkovich, Fuzzy control, Menlo Park, Calif., Addison-Wesley, 1998.

[33] F. Previdi and E. Carpanzano, Design of a gain scheduling controller for Knee-Joint angle control by using functional electrical stimulation, Ieee Transactions on Control Systems Technology 11(3) (2003), 310-324.

[34] R. Riener and T. Fuhr, Patient-driven control of FESsupported standing up: A simulation study, IEEE Trans Rehabil Eng 6(2)(1998), 113-124.

[35] A. Stefanovska, L. Vodovnik, N. Gros, S. Rebersek and R. Acimovic-Janezic, FES and spasticity, IEEE Trans Biomed Eng 36(7) (1989), 738-745.

[36] A. Trnkoczy, Variability of electrically evoked muscle contractions with special regard to closed-loop controlled orthosis, Annals of Biomedical Engineering 2(2) (1974), 226-238

[37] Y. Uno, M. Kawato and R. Suzuki, Formation and control of optimal trajectory in human multijoint arm movement minimum torque-change model, Biological Cybernetics 61(2) (1989), 89-101.

[38] P.H. Veltink, Control of FES-induced cyclical movements of the lower leg, Med Biol Eng Comput 29(6) (1991), NS8-N12.

[39] P.H. Veltink and A.J. Mulder, Optimization criteria for control of FES, Restoration of Walking for Paraplegics. Recent advancements and trends, A. Pedotti and M. Ferrarin, Milano, IOS Press, (1992), 277-281.

[40] M.M. Williamson, Exploiting natural dynamics in Robot control, Cybernetics and systems research, Vienna, Austrian Society for Cybernetic Studies, 1998.

[41] D.A. Winter, Biomechanics and motor control of human movement, Hoboken, New Jersey, John Wiley \& Sons, 2005.

[42] M. Zhuang and D.P. Atherton, Automatic tuning of optimum PID controllers, Control Theory and Applications, IEE Proceedings D [see also IEE Proceedings-Control Theory and Applications] 140(3) (1993), 216-224.

[43] J.G. Ziegler and N.B. Nichols, Optimum setting for PID controllers', Transactions of ASM 64 759-768. 

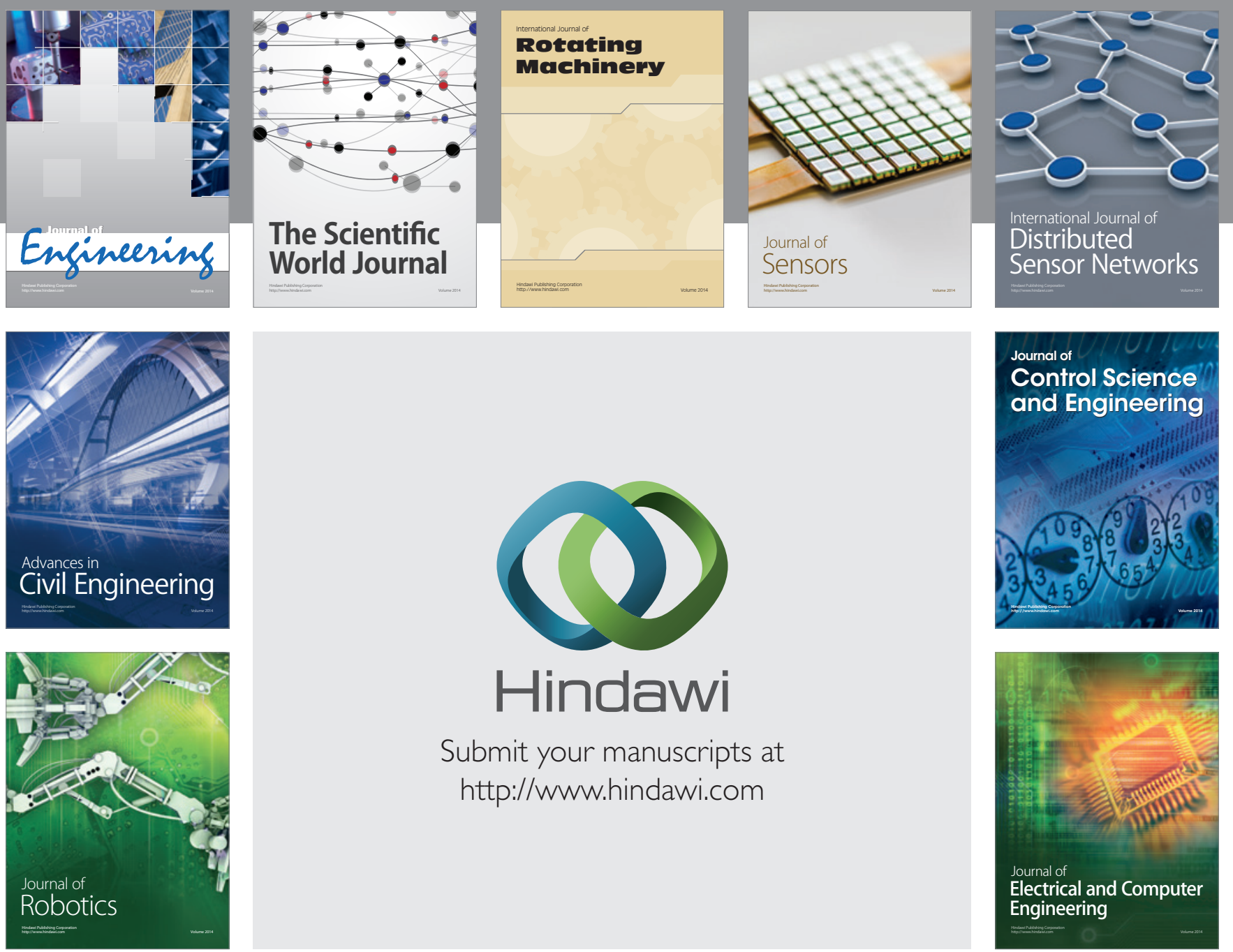

Submit your manuscripts at

http://www.hindawi.com
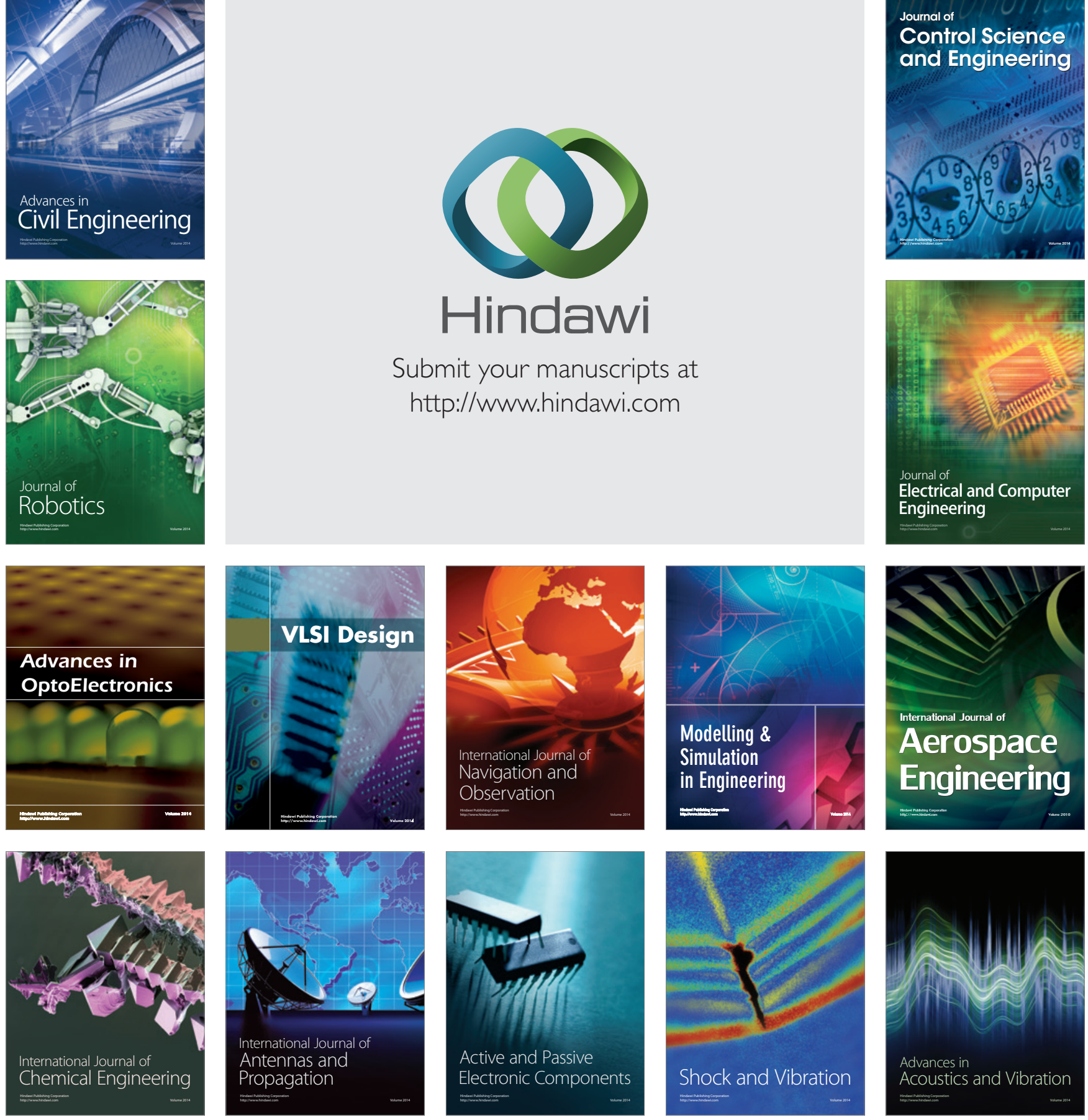